Anal. Theory Appl.

Vol. 28, No. 1 (2012), 95-100

\title{
A MATHEMATICAL PROOF OF A PROBABILISTIC MODEL OF HARDY'S INEQUALITY
}

\author{
Prateek K \\ (Taleigao Plateau Goa University, India)
}

Received June 23, 2010; Revised Feb. 14, 2012

\begin{abstract}
In this paper using an argument from [1] , we prove one of the probabilistic version of Hardy's inequality.
\end{abstract}

Key words: random variables, uniform partition, Hardy's inequality

AMS (2010) subject classification: 11K99, 11P55

\section{Introduction}

Hardy's inequality is defined as for a constant $c>0$, we have

$$
\sum_{n=1}^{\infty} \frac{|\hat{f}(n)|}{n} \leq c\|f\|_{1}
$$

for all functions $f \in L^{1}([0,2 \pi))$ with $\hat{f}(n)=0$ for $n<0$. This inequality is not true for all functions $f \in L^{1}([0,2 \pi))$, which can be seen by letting $f$ to be the Fejér kernel of order $N$ for large enough $N$.

When McGehee, Pigno and Smith ${ }^{[3]}$ proved the Littlewood conjecture, many questions were asked of how Hardy's inequality can be generalized for all functions $f \in L^{1}([0,2 \pi))$. For instance, one of the expected generalizations is the following:

$$
\sum_{n>0} \frac{\hat{f}(n) \mid}{n} \leq c\|f\|_{1}+c \sum_{n>0} \frac{|\hat{f}(-n)|}{n}, \quad f \in L^{1}([0,2 \pi)),
$$

where $c>0$ is an absolute constant.

In this paper, we prove one version of Hardy's inequality for functions whose Fourier coefficients $\hat{f}(n)$ are random variables on $(0,1)$ for $n>0$ without conditions on $\hat{f}(n)$ for $n<0$. 
In my proof use a technique that was motivated by Körner ${ }^{[1]}$, who used this technique in a different problem to modify a result of Byrnes (see [1]).

In the sequel, $[0,2 \pi)$ denotes the unit circle, $L^{1}([0,2 \pi)$ the space of integrable functions on $[0,2 \pi), \mu$ the Lebesgue measure, and $B_{j}$ the set of integers in the interval $\left[4^{j-1}, 4^{j}\right)$.

\section{Basic Lemmas}

In this section, I am going to prove some basic lemmas required for our purpose.

Lemma 2.1. Let $X_{1}, X_{2}, \cdots, X_{N}$ be independent random variables such that

$$
\left|X_{j}\right| \leq 1 \quad \text { for each } \quad j, 1 \leq j \leq N,
$$

and write

$$
S_{N}=X_{1}+X_{2}+\cdots+X_{N}
$$

Then, for any $\lambda>0$,

$$
\operatorname{Pr}\left(\left|S_{N}-E S_{N}\right| \geq \lambda\right) \leq 4 \exp \left(-\frac{\lambda^{2}}{100 N}\right) .
$$

For the proof, see [4, p.398].

The idea of the following proof is due to Köner ${ }^{[1]}$. The statement of the lemma was observed by Kahane ${ }^{[2]}$ without proof.

Lemma 2.2. Let $\left(r_{k}\right)$ be a sequence of independent, zero mean random variables defined on the interval $(0,1)$ with $\left|r_{k}\right| \leq 1$ for all $k$. Let

$$
f_{n}(\theta, t)=\sum_{p=1}^{n} r_{p}(t) e^{i p \theta} \quad \text { for } \quad t \in(0,1) \quad \text { and } \quad \theta \in[0,2 \pi) .
$$

Then for $n \geq 27$ and $\lambda \geq 2 \times 2$,

$$
\mu\left(\left\{t: \sup _{\theta}\left|f_{n}(\theta, t)\right| \geq \lambda \sqrt{n \log n}\right\}\right) \leq 4 n^{2-\frac{\lambda^{2}}{400}} .
$$

Proof. By applying Lemma 2.1, we find that for fixed $\theta \in[0,2 \pi)$,

$$
\mu\left(\left\{t: \sup _{\theta}\left|f_{n}(\theta, t)\right| \geq \lambda \sqrt{n \log n}\right\}\right) \leq 4 n^{2-\frac{\lambda^{2}}{100}} .
$$

Let $\left(\theta_{k}\right)_{k=1}^{n^{2}}$ be a uniform partition of the unit circle. For fixed $t \in(0,1)$ and $\theta_{k} \in[0,2 \pi)$ and for all $\theta$ with $\left|\theta-\theta_{k}\right| \leq 2 \pi / n^{2}$, we have

$$
\left|f_{n}(\theta, t)-f_{n}\left(\theta_{k}, t\right)\right| \leq \sum_{p=1}^{n}\left|r_{p}(t)\right|\left|e^{i p \theta}-e^{i p \theta_{k}}\right| \leq 2 \sum_{p=1}^{n} \frac{2 \pi}{n^{2}} p=\frac{2 \pi(n+1)}{n} .
$$


Lemma 2.3. There exists a set $\subset(0,1)$ of measure 1 such that whenever $t \in B$ there exists an index $k_{t}$ with the property that

$$
\sup _{\theta}\left|g_{j}(\theta, t)\right| \geq 60 \sqrt{j 4^{-j}}, \quad \forall j \geq k_{t} .
$$

Proof. Let

$$
M_{k}=\bigcup_{j=k}^{\infty} A_{j} \quad \text { also } \quad M=\bigcap_{k=1}^{\infty} M_{k} .
$$

Thus,

$$
\mu(M)=\mu\left(\bigcap_{k=1}^{\infty} M_{k}\right) \leq \mu\left(M_{k}\right)
$$

for all $k \geq 1$, i.e.,

$$
\mu(M) \leq \mu\left(\left(\bigcup_{j=k}^{\infty} A_{j}\right) \leq \sum_{j=k}^{\infty} \mu\left(A_{j}\right)\right.
$$

for all $k \geq 1$. As

$$
\mu\left(A_{j}\right) \leq 8 \times 4^{-j / 4} \quad \text { and } \quad \sum 4^{-j / 4}<\infty,
$$

hence

$$
\mu(M) \leq \sum_{j=k}^{\infty} \mu\left(A_{j}\right) \rightarrow 0 \quad \text { as } \quad k \rightarrow \infty .
$$

Thus, $\mu(M)=0$. Putting $B=M^{C}$, the lemma is proved.

\section{Main Result}

In this section, we prove the probabilistic version of Hardy's inequality, which is main contribution in this paper.

Thus, for fixed $t$ and $\theta_{k}$ and for all $\theta$ such that $\left|\theta-\theta_{k}\right| \leq 2 \pi / n^{2}$, we have

$$
\left|f_{n}(\theta, t)\right| \leq \frac{2 \pi(n+1)}{n}+\left|f_{n}\left(\theta_{k}, t\right)\right|,
$$

and consequently

$$
\sup _{\left|\theta-\theta_{k}\right| \leq \pi / n^{2}}\left|f_{n}(\theta, t)\right| \leq \frac{2 \pi(n+1)}{n}+\left|f_{n}\left(\theta_{k}, t\right)\right| .
$$

But on a set (of $t$ ) of measure $\geq 1-4 n^{2-\frac{\lambda^{2}}{100}}$ we have for each $\theta_{k}$

$$
\mid f_{n}\left(\theta_{k}, t\right) \leq \lambda \sqrt{n \log n}
$$


Therefore, for any particular $\theta_{k}$ we have on a set (of $t$ ) of measure $\geq 1-4 n^{2-\frac{\lambda^{2}}{100}}$,

$$
\sup _{\theta-\theta_{k} \mid \leq 2 \pi / n^{2}}\left|f_{n}(\theta, t)\right| \leq \frac{2 \pi(n+1)}{n}+\lambda \sqrt{n \log n} .
$$

Since the set

$$
\left\{t: \sup _{\theta}\left|f_{n}(\theta, t)\right| \geq \frac{2 \pi(n+1)}{n}+\lambda \sqrt{n \log n}\right\}
$$

is contained in the set

$$
\bigcup_{k=1}^{n^{2}}\left\{t: \sup _{\left|\theta-\theta_{k}\right| \leq 2 \pi / n^{2}}\left|f_{n}(\theta, t)\right| \leq \frac{2 \pi(n+1)}{n}+\lambda \sqrt{n \log n}\right\},
$$

we must have

$$
\mu\left(\left\{t: \sup _{\theta}\left|f_{n}(\theta, t)\right| \geq \frac{2 \pi(n+1)}{n}+\lambda \sqrt{n \log n}\right\}\right) \leq \sum_{p=1}^{n^{2}} 4 n^{-\frac{\lambda^{2}}{100}}=4 n^{2-\frac{\lambda^{2}}{100}}
$$

If $\lambda \geq \sqrt{2}$ and $n \geq 27$, we have

$$
\frac{2 \pi(n+1)}{n} \leq \lambda \sqrt{n \log n}
$$

hence it follows that

$$
\mu\left(\left\{t: \sup _{\theta}\left|f_{n}(\theta, t)\right| \geq 2 \lambda \sqrt{n \log n}\right\}\right) \leq 4 n^{2-\frac{\lambda^{2}}{100}} .
$$

On replacing $2 \lambda$ by $\lambda$

$$
\mu\left(\left\{t: \sup _{\theta}\left|f_{n}(\theta, t)\right| \leq \lambda \sqrt{n \log n}\right\}\right) \leq 4 n^{2-\frac{\lambda^{2}}{400}} .
$$

whenever $\lambda \geq 2 \sqrt{2}$ and $n \geq 27$.

Thus, by letting

$$
g_{j}(\theta, t)=\sum_{n \in B_{j}} r_{n}(t) e^{i n \theta},
$$

where $B_{j}$ denotes the set of integers in the interval $\left[4^{j-1}, 4^{j}\right)$, we see that

$$
\mu\left(\left\{t: \sup _{\theta}\left|g_{j}(\theta, t)\right| \geq 2 \lambda \sqrt{j 4^{-j}}\right\}\right) \leq 4\left(\frac{3}{4}\right)^{2-\frac{\lambda^{2}}{400}}\left(4^{\left(2-\lambda^{2}\right) \frac{j}{400}}\right.
$$

for all $\lambda \geq 2 \sqrt{2}$ and $j \geq 4$. By choosing $\lambda=30$, we see that

$$
\mu\left(A_{j}\right) \leq 8 \times 4^{-j / 4} \quad \text { for } \quad j \geq 4,
$$


where

$$
A_{j}=\left\{t: \sup _{\theta}\left|g_{j}(\theta, t)\right| \geq 60 \sqrt{j 4^{-j}}\right\} .
$$

Theorem 3.1. Let $\left(r_{k}\right)$ be a sequence of independent, zero mean random variables on the interval $(0,1)$, with $\left|r_{k}\right|=1$ for all $k$. Then there exists a set $S \subset(0,1)$ of measure 1 such that

$$
\sum_{n=1}^{\infty} \frac{\hat{f}(n) \mid}{n} \leq c\|f\|_{1}
$$

for all functions $f \in L^{1}([0,2 \pi))$ satisfying the condition

$$
\hat{f}(n) \overline{r_{n}(t)} \geq 0, \quad \text { for all } \quad n>0 .
$$

Proof. Let $t \in B$ be fixed. It suffices to prove the result for all trigonometric polynomials $f$ with

$$
\hat{f}(n) \overline{r_{n}(t)} \geq 0, \quad \text { for all } \quad n>0 .
$$

Thus, let $f$ be a trigonometric polynomials with $\hat{f}(n) \overline{r_{n}(t)} \geq 0$

$$
F(\theta)=\sum_{j=1}^{\infty} g_{j}(\theta, t) \text {. }
$$

It is clear from the definition of $g_{j}$ that

$$
\hat{F}(n)=\frac{r_{n}(t)}{4^{j}}, \quad \text { for } \quad n>0,
$$

where $j$ is the unique index such that $n \in B_{j}$. Also, we see that

$$
\hat{F}(n)=0, \quad \text { for } \quad n \leq 0 .
$$

Since $t \in B$, we conclude that

$$
\sum_{j=1}^{\infty} \sup _{\theta \in[0,2 \pi)}\left|g_{j}(\theta, t)\right|:=K<\infty .
$$

Therefore, $F$ is a bounded function on the circle with $\|F\|_{\infty} \leq K$.

Now, we apply a standard duality argument to obtain

$$
\begin{aligned}
K\|f\|_{1} & :=\|F\|_{\infty}\|f\|_{1} \geq \frac{1}{2 \pi}\left|\int_{0}^{2 \pi} f(\theta) \overline{F(\theta)} \mathrm{d} \theta\right|=\left|\sum_{n \in \mathbf{Z}} \hat{f}(n) \overline{\hat{F}(n)}\right| \\
& =\left|\hat{f}(0) \overline{\hat{F}(0)}+\sum_{n>0} \hat{f}(n) \overline{\hat{F}(n)}\right|, \\
K\|f\|_{1} & =\left|\sum_{n>0} \hat{f}(n) \hat{\hat{F}(n)}\right|-|\hat{f}(0)||\hat{F}(0)|,
\end{aligned}
$$


hence,

$$
2\|f\|_{1}\|F\|_{\infty} \geq\left|\sum_{j=1}^{\infty} \sum_{n \in B_{j}} \hat{f}(n) \overline{\hat{F}(n)}\right|=\left|\sum_{j=1}^{\infty} \sum_{n \in B_{j}} \hat{f}(n) \frac{\overline{r_{n}(t)}}{4^{j}}\right| \geq \frac{1}{4} \sum_{j=1}^{\infty} \sum_{n \in B_{j}} \frac{|\hat{f}(n)|}{n} .
$$

Thus we have proven the above theorem for $c=8 k$.

\section{References}

[1] Körner, T. W., On a Polynomials of Byrnes, BUl1. London Math. Soc., 12(1980), 219-224.

[2] Kanane, J. -P., Sur Les Polynömer á coefficients unimodulaires, Bull. London Math. Soc., 12(1980), 321-342.

[3] McGehee, O. C., Pigno, L. and Smith, B., Hardy's Inequality and the $L^{1}$ Norm of Exponential Sums, Annals Math., 113(1981), 613-618.

[4] Rënyl, A., Probability Theory, North-Holland (Amsterdam, 1970).

[5] Smith, B., Two Trigonometric Designs, ISNM 65 General Inequalities, 3 Birkhauser (Basel, 1983), 141-148.

Taleigao Plateau Goa University

Panaji-Goa

India

E-mail: kvprateek@gmail.com 